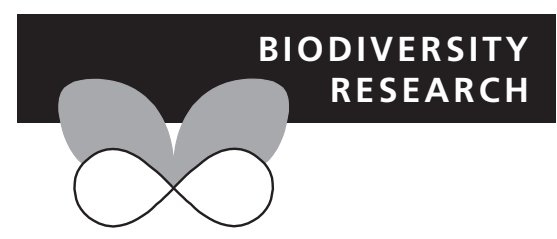

\title{
Quantitative comparison and selection of home range metrics for telemetry data
}

Graeme S. Cumming $^{1 *}$ and Daniel Cornélis ${ }^{2}$

${ }^{1}$ Percy FitzPatrick Institute, DST/NRF

Centre of Excellence, University of Cape Town, Rondebosch, Cape Town, 7701, South Africa, ${ }^{2}$ CIRAD, Animal et Gestion Intégrée des Risques, Campus de Baillarguet, 34398, Montpellier, France

*Correspondence: Graeme S. Cumming, Percy FitzPatrick Institute, DST/NRF Centre of Excellence, University of Cape Town, Rondebosch, Cape Town 7701, South Africa. E-mail: graeme.cumming@uct.ac.za.

\section{ABSTRACT}

Aim Home range (HR) metrics are widely used in ecology and conservation, but the quantitative basis for choosing and parameterizing metrics is weak. Home range estimates are ecological and statistical hypotheses that must balance type I and type II errors. Here, we present and test a new approach to fine-tuning and comparing HR estimates using the area under the curve (AUC) statistic.

Location Test data are taken from telemetry studies of 44 individual ducks in southern Africa and nine buffaloes in southern and western Africa.

Methods We use a meta-analysis of AUC statistics to compare the performance of four standard HR metrics on data from 44 ducks (two species) and nine African buffaloes.

Results The AUC method emerges as a useful and accessible statistical tool. It captures clear differences between HR estimators as well as providing a way of fine-tuning parameters for an individual HR estimate. Code to run the HR AUC analyses in $\mathrm{R}$ is provided. As argued by others, we found that kernel density estimators offer the best combination of ecological and statistical validity, while estimators that use minimum convex polygons at any stage of the algorithm perform poorly and should be avoided.

Main conclusions The AUC statistic provides a readily implementable and straightforward approach to comparing different HR metrics and to selecting parameters for individual metrics. It thus offers a valuable tool for conservation efforts that seek to define HRs for species or populations. The use of the AUC in this new context further contributes to solidifying the interface between species occurrence models and HR estimators.

\section{Keywords}

Argos, home range, kernel, LoCoH, movement ecology, PTT, ROC plot, species occurrence model, telemetry.

\section{INTRODUCTION}

Understanding why different elements of biodiversity occur where they do is fundamental to conservation biogeography (Richardson \& Whittaker, 2010). Biodiversity ranges from genes to communities and occurs from fine to broad scales. At fine scales and the level of a single individual or population, home range (HR) metrics quantify the extent of habitat that an individual or population of animal uses (e.g. Kelt \& Van Vuren, 2001; Valeix et al., 2009). Home range analysis is thus an important first step in understanding the mecha- nisms and spatial relationships that underlie habitat choice and the responses of animals to environmental change (e.g. Akcakaya, 2000; Thomas \& Taylor, 2006).

At broader scales, species occurrence modelling has become a prominent research area in conservation biogeography (Richardson \& Whittaker, 2010). Home range metrics are closely related to species occurrence models in that they provide important insights into the mechanisms - such as dispersal, choice of foraging habitat, and the use of complementary areas for different purposes - that underpin and explain species occurrences (e.g. see Guisan et al., 2006). 
Home range metrics have, however, proceeded more or less in isolation from species distribution models. In seeking multi-scale and multi-level explanations for ecological phenomena, there is a clear need to better integrate individualand species-level models to provide stronger predictions about biodiversity change (Cumming, 2007).

Home range metrics have become more sophisticated as computing and telemetry technologies have advanced and as the variety of applications of HR estimators has increased. Early analyses of both HRs and species occurrences delineated boundaries using a minimum convex polygon (MCP) (Mohr, 1947), which is the smallest area that includes all points and has no acute angles on its external boundary. MCPs include all of the animal resightings in a data set (or 95\% in a 'peeled' MCP; Schoener 1981), but tend to heavily overestimate the extent of HRs or species ranges and ignore the distribution of space use within their borders. Although estimators that simply determine a HR boundary may be useful in some cases (e.g. when the number of relocations is very low), HR estimators that take into account the utilization distribution of space use (and thus allow distinction between core and peripheral areas) are clearly more useful.

As the weaknesses of MCP approaches have become evident (Seaman et al., 1999; Burgman \& Fox, 2003; Laver \& Kelly, 2008), a variety of more rigorous methods for estimating HRs has been developed (reviewed in Börger et al. 2008). Curiously enough, however, there is no single accepted statistic for comparing the quantitative estimates that are produced for the same data set by different HR metrics. The development of such statistics has been fundamental to the progress of species occurrence modelling (e.g. Fielding \& Bell, 1997; Cumming, 2000, 2002; Franklin, 2010). Although several possibilities have been proposed, including maximum likelihood analysis and the comparison of volumes of intersection (e.g. Horne \& Garton, 2006; Lichti \& Swihart, 2011), none of the current alternatives has the three desirable qualities of being (1) straightforward to quantify and interpret, (2) statistically rigorous and (3) free of assumptions about the underlying statistical distribution of points in space.

In this paper, we contribute to the quantitative basis for the comparison of HR metrics. We first lay down a set of criteria (both qualitative and quantitative) for consideration when selecting a HR metric. We then explain how a widely used statistic, the area under the curve (AUC) of a threshold receiver operating curve (ROC), can be used to compare HR metrics. We use our new approach to evaluate the performance of four commonly used metrics on two sets of GPS satellite telemetry data derived from 44 individual birds of two different species (Red-billed Teal, Anas erythrorhyncha; and Egyptian Geese, Alopochen aegyptiacus, both in family Anatidae) and nine individual buffalo (Syncerus caffer). As our analysis demonstrates, AUC statistics are useful for comparing between estimators and for fine-tuning parameter choices for any single metric. Their relevance in this context further supports the desirability of exploring the nexus between species occurrence models and HR estimates.

\section{CRITERIA FOR SELECTING HOME RANGE METRICS}

The use of a given HR metric must be dictated first and foremost by ecological considerations. The selection of ecologically relevant metrics is well discussed by other authors and as it is not our primary target in this paper, we refer the interested reader to Laver \& Kelly (2008) or Kie et al. (2010) for further detail. A good general discussion of sources of 'biotic error' in spatial estimation routines can be found in Fielding \& Bell (1997).

In statistical terms, a $\mathrm{HR}$ algorithm behaves as a spatial (and sometimes a temporal) classifier that must assign each location in a chosen area a probability of belonging to the HR. In solving this problem, both type I and type II errors (in this context, more easily thought of as errors of inclusion or comission and exclusion or omission) are important. Kenward et al. (2001) suggested that 'The solution must be to minimize the risk of type I errors by a priori selection of estimators'. More recently, Getz et al. (2007) have stated that 'Obviously, a method that produces both smaller type I and type II errors than another is preferred, provided the method is not computationally difficult to implement'. Although this statement improves on the earlier focus on type I errors, it ignores the point that the accuracy of a spatial classifier rests on a trade-off between two antagonistic sources of error. Increased type I errors will generally result in decreased type II errors and vice versa. A statistically sound HR metric will include the majority of resightings within the $\mathrm{HR}$ and exclude an ecologically defensible number of non-resightings from the HR.

In practical terms, HR metrics should be feasible to implement using standard technologies. They should also be robust to unusual data sets. For instance, 'holes' may reflect estimation errors or genuine landscape pattern (e.g. a human settlement occurs inside a protected area); and clusters of points that are surrounded by other clusters (e.g. littoral animals, such as sandpipers, that may use an island in the middle of a small lake as well as the lakeshore but not the water in between) should be detected.

Lastly, there is an increasing recognition in animal movement ecology that habitat use by animals is multi-scale (Boyce, 2006; Mayor et al., 2009). A good HR estimator will facilitate the characterization and description of multi-scale habitat use, capturing not only core versus periphery but also different 'islands' of frequently used habitat at different scales. These will often be areas used during different seasons or for different activities, such as breeding and foraging.

To summarize, we propose that a priori, a quantitatively sound HR estimator will: (1) optimize the trade-off between errors of omission and commission; (2) distinguish between high- and low-use areas (i.e. indicate core and periphery); (3) be suitably sensitive to landscape features that constrain or alter animal movements, such as roads or fencelines and be robust to unusual data, in the sense that the estimator should deal appropriately with examples in which the topology 
of habitat use is not a single, smooth polygon; (4) offer some indication of stratification within the broader $\mathrm{HR}$, and ideally capture elements of multi-scale HR structure; and (5) be computationally efficient enough to implement on a laptop or desktop computer for large data sets.

\section{STATISTICAL COMPARISON OF HOME RANGE METRICS}

A useful parallel to the HR estimator problem - and indeed, a solution to what is virtually the same problem - comes from approaches to predicting species occurrences from environmental variables. Most measures of the goodnessof-fit of species occurrence models are based on an error matrix ('confusion matrix', as summarized in Appendix S1 in supporting information) that describes the numbers of true and false positives and negatives included at a given probability value (Cumming, 2000, 2009). Quantitative classifiers seek to optimize the trade-offs between entries in the four cells of the confusion matrix, minimizing error and maximizing accuracy (Fielding \& Bell, 1997).

Integrating the plot of sensitivity against 1-specificity over a range of probability thresholds (or for HR metrics, isopleths) produces a measure termed the AUC of the threshold ROC. The AUC provides an indication of the overall fit of the model to the data and hence offers a single metric of the goodness-of-fit (Fielding \& Bell, 1997; Cumming, 2000). The AUC has been well documented and will be familiar to many readers but has not previously been applied to the comparison of HR estimators.

It is important to note that we are not proposing that the AUC be applied as an overall measure of goodness-of-fit of a HR metric. The AUC does not answer the question of whether a substantially different HR might have been estimated if a different set of resightings were to be collected from the same individual. Assessment of the 'absolute' goodness-of-fit of a model is primarily a question for information-theoretic statistics such as Akaike's information criterion, combined with bootstrapping and jackknifing approaches, rather than the AUC (Vaughan \& Ormerod, 2005).

What we are proposing is that the AUC is an ideal relative metric for comparing the results of different estimators when applied to the same data set. Used in this way, any biases inherent in the data are held constant while different HR metrics, and the parameters (such as smoothing values) that influence their outputs, are objectively and quantitatively compared. For instance, even though true absences can be difficult to define with typical telemetry data sets, if the same assumption about true absences holds for all competing HR estimates (or parameters) then the best estimate will still have the higher AUC.

Published concerns over AUCs (e.g. Peterson et al., 2008) arise primarily from the fact that the AUC is scale dependent. It is influenced by prevalence, which is the ratio of positive to negative values (in the context of HRs, resightings to non-resightings) in the data and hence by both the grain and extent of analysis (Cumming, 2000). For example, the AUC of a species occurrence model can be artificially inflated (without adding any ecological meaning) by increasing the extent of the analysis to include unsampled areas without species presences (Cumming, 2000). Related statistics such as kappa, however, suffer from the same weakness. In fact, as nearly all frequentist statistics are influenced by sample size and correspondingly by the grain and extent of sampling, virtually any statistic that is applied to spatial data and does not explicitly correct for both grain and extent will be influenced by prevalence (Cumming, 2009).

The AUC has also been criticized for not providing a definite cut-off point for inclusion or exclusion (species present or species absent). Presence/absence delineation, when needed, is more readily determined by the use of simple heuristics, such as selecting as a threshold the probability value that includes $95 \%$ of known resightings (i.e. the 95\% isopleth). This approach is already the standard approach for determining which points fall inside or outside a HR estimate; and if necessary, the choice of a different isopleth (e.g. a $50 \%$ isopleth for 'core area' analysis) can be justified by arguing its ecological meaning.

One further limit on the AUC must be mentioned. This applies to data sets in which multiple resightings occur from the same location (e.g. animals observed repeatedly near the same burrow or roosting site). As the ROC plot traditionally uses binary (presence/absence) data to calculate specificity and sensitivity, most implementations of the AUC will treat cells as simply being occupied or unoccupied during estimation of goodness-of-fit. This means that the additional capabilities offered by algorithms (such as kernel estimators) that capture variations in point density within each grid cell will be ignored during AUC-based comparisons. In this paper, this consideration is not important, but it would arise in the comparison of kernel-based methods to approaches that do not weight repeatedly resampled locations more highly than singly sampled locations.

\section{METHODS: AN AUC-BASED COMPARISON OF HOME RANGE METRICS}

We compared a set of state-of-the art HR metrics [Appendix S2; see also Worton (1989, 1995), Kenward et al. (2001), Getz \& Wilmers (2004), Calenge (2006), Benhamou \& Cornelis (2010), and Benhamou (2011)] using the AUC statistic and data from 44 GPS-Argos-tracked birds of two Afrotropical species, Red-billed Teal $(n=20)$ and Egyptian Geese $(n=24)$. To further diversify the comparison, we also compared the data for nine individually GPS-UHF-tracked African buffalo. The numbers of resightings in each individual test data set ranged from 72 to 9697 . Further details on the data sets are provided in Appendix S3.

The results from any AUC-based assessment of a spatial model are scale dependent. We thus ensured that all calculations for any single data set were performed using the same 
resighting data, at the same grain and extent and on genuinely comparable outputs. As some metrics (e.g. the kernel density estimator) generate raster files and as the assessment as a whole is easier to programme using raster data, all HR estimates (i.e. whether the output was raster or vector) were converted to raster files at identical grain and extent for the calculation of AUCs.

GIS and statistical analyses were run in the software packages Arcview, R, Free Pascal, and Matlab. Example code for $\mathrm{R}$ and Matlab routines is provided in Appendix S4. In $\mathrm{R}$, we used the standard implementations offered in the adehabitat/ adehabitatHR package as developed by Calenge (2006) and including the LoCoH algorithm developed by Getz \& Wilmers (2004); Getz et al. (2007). The movement-based kernel density eEstimator (MKDE) (Benhamou \& Cornelis, 2010) was computed using Pascal (duck data) and the adehabitatHR package (R Software, buffalo data). AUC estimation was performed independently in $\mathrm{R}$ (buffalo) and Matlab (ducks). For the duck data, $\mathrm{R}$ routines were called from within Matlab, allowing the use of Matlab functions for other data manipulations (e.g. reprojection and the estimation of isopleth extents) and timing. The use of Matlab was not essential, but it was the programming environment in which other analyses of the duck data were already being carried out. Technological details and processing times for different algorithms are presented in Appendix S5.

A single base raster map for each data set was created manually in ArcView at 0.005-degree resolution (ducks; i.e. c. $556 \mathrm{~m}$ ) or $100 \mathrm{~m}$ (buffalo) and at an extent at which each point was at least 0.1 degrees (ducks; c. 11,000 m) or $5000 \mathrm{~m}$ (buffalo) away from the edge. Although for less extensive data sets a finer resolution would be both feasible and biologically preferable, we wanted to keep everything as consistent as possible when comparing between different methods and data sets. The base map was used for each estimator to determine both grain and extent, thus removing variation in scale and prevalence as potential confounding variables in the comparison of different estimators.

For the MKDE estimator as run on the duck data, a raster map was generated in UTM coordinates, and the results were reprojected into geographic coordinates before AUC analysis. This was necessary because the MKDE routine uses UTM coordinates but the duck data in many cases span several UTM zones. The reprojection process meant that an exact match in the total number of cells was not always possible. However, prevalence (i.e. total number of cells scored as resightings as a ratio of the total number of cells in the raster file) for all MKDE analyses differed from prevalence in other analyses by a mean of just 0.0003 and was within 0.001 of the prevalence for other estimators in all cases. Differences of this magnitude affect only the fourth or fifth decimal point of the AUC and hence are not sufficiently large to influence the outcome of the comparison. The data for each individual buffalo, by contrast, fell within a single UTM tile.

We implemented each of the four HR estimators for all 44 ducks and nine buffalo, with three exceptions: (1) HR estimation for ClustHR was limited by the demands of processing time to data from 25 ducks and seven buffalo data sets and (2) owing to minimum sample size requirements for the MKDE metric, we used data for only 38 ducks for this estimator.

\section{RESULTS}

For the duck data, a Kruskal-Wallis (nonparametric) ANOVA on AUC results across the different estimators gave a strong significant difference between the four HR estimators (chi-squared $=77.15, \quad P<0.00$ ). Visual inspection of the results (Fig. 1) and the means and deviations for each result set (Fig. 2) suggested that the two MCP-based methods, Lo$\mathrm{CoH}$ and ClustHR, were weaker than the other two methods. A Kruskal-Wallis ANOVA on the LKDE and MKDE results for the duck data did not yield a significant difference (chisquared $=0.95, P<0.32$ ). The two kernel-based methods thus perform similarly on the duck data sets and significantly better than the two MCP-based methods.

For the buffalo data, the Kruskal-Wallis ANOVA test identified a significant difference between estimators (chisquared $=28.8, P<0.00$ ) with the LoCoH algorithm performing notably worse than the other estimators (Fig. 2). With $\mathrm{LoCoH}$ excluded there was still a significant difference in the other three estimators using the same test (chisquared $=16, P<0.00$ ). In this instance, however, the ClustHR and LKDE estimators did not differ significantly (chisquared $=1, P<0.30$ ), and the MKDE estimator performed better than the LKDE estimator (Kruskal-Wallis ANOVA, chi-squared $=9.83, P<0.00)$.

Differences in the total HR area estimated by different algorithms were substantial in some cases, particularly for the more extensive data sets. The exact nature of the differences depended on both the metric and the isopleth (Fig. 3). For the duck data, for example, at the $95 \%$ isopleth the area described by $\mathrm{LoCoH}$ algorithm was an average of $14 \%$ smaller than that of the kernel density estimator. At the $50 \%$ isopleth, however, the average HR area estimated by the $\mathrm{LoCoH}$ algorithm across our test data sets was about 2.5\% larger than that of the LKDE or MKDE estimates. Given that the average area of the extent over which HR estimation was undertaken was $46,500 \mathrm{~km}^{2}$, these differences translate into average areas of 6510 and $1163 \mathrm{~km}^{2}$, respectively. Differences in the degree to which different estimators identified finerscale clusters in the data were also evident in many of the duck data sets (Fig. 4).

\section{DISCUSSION}

Our results provide a strong demonstration of the value of comparing HR metrics using a method that integrates the error trade-off across the range of probabilities or densities assigned by the classifier, rather than evaluating the statistical performance of metrics based on the area of a single isopleth or a combination of subjective criteria. The AUC statistic 
(a)

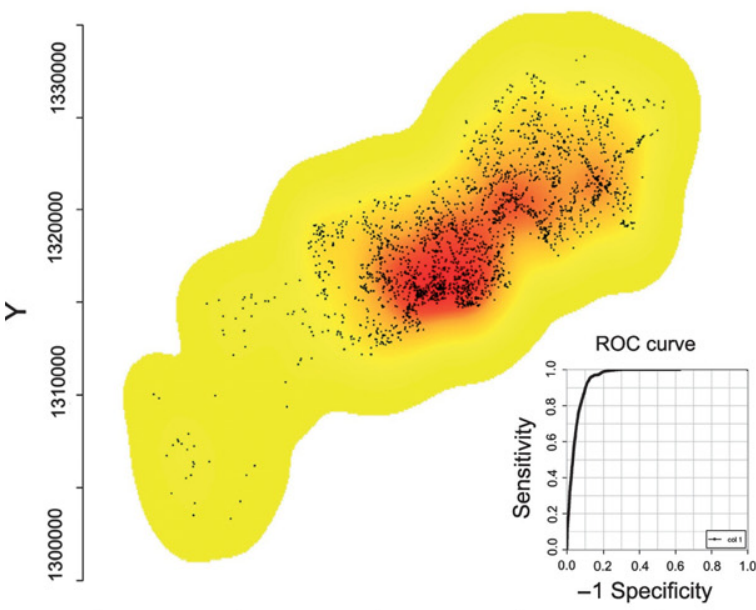

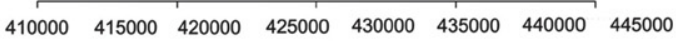

$\mathrm{X}$

(c)

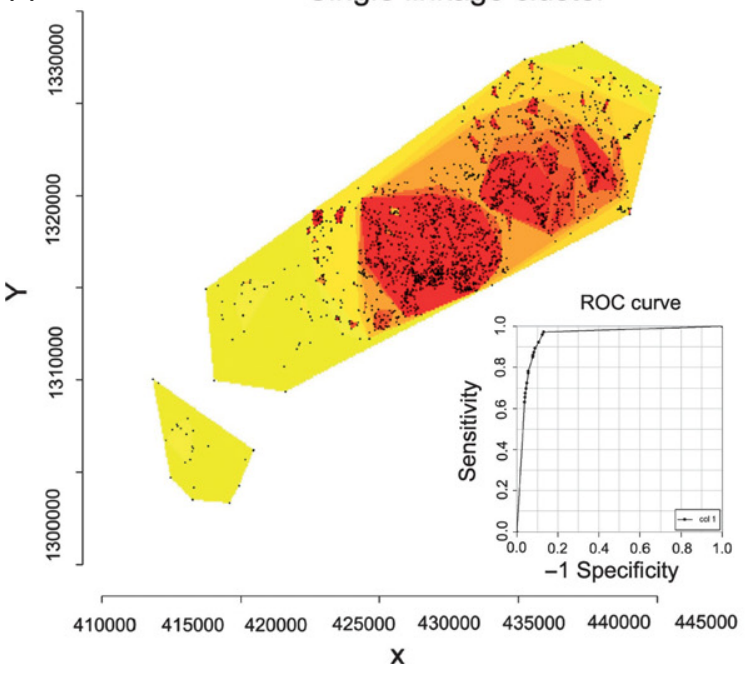

(b)

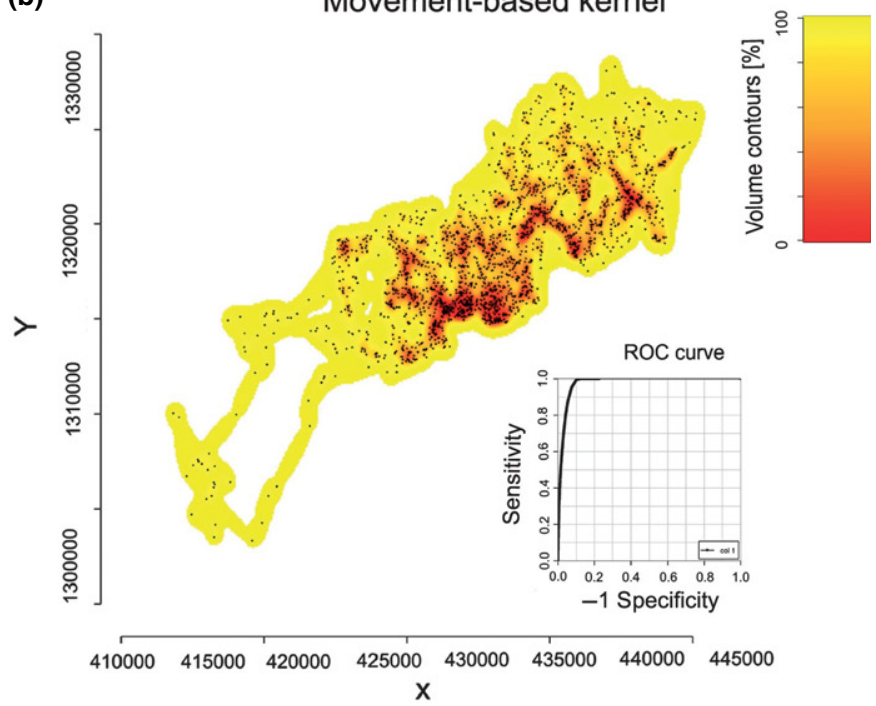

(d)

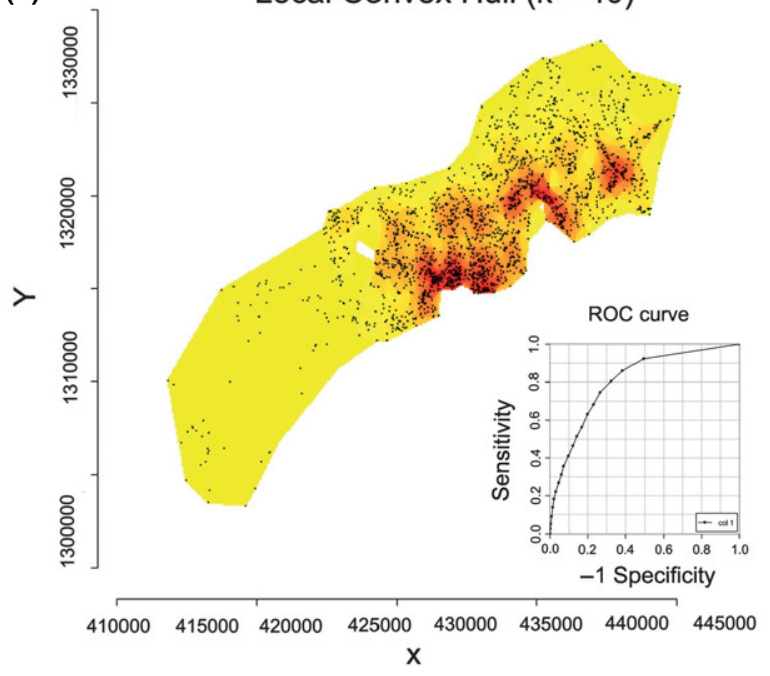

Figure 1 Example data set for a single buffalo, showing differences in home range estimates produced by different estimators considered in this paper: (a) LKDE, (b) movement-based kernel density eEstimator (MKDE), (c) ClustHR and (d) LoCoH. Estimator names and methods are explained in detail in Appendix S2.

provides useful insights both when comparing HR estimators and when exploring the implications of different choices of smoothing parameters and algorithms. AUCs also avoid assumptions about the statistical distribution of resightings and remove the need for creating an additional 'true' validation data set (as in Lichti \& Swihart, 2011) when testing a new HR metric. Each of the HR estimators considered in this paper has its own strengths and weaknesses (Table 1), and the user must make an informed choice of metric based first and foremost on the philosophical and ecological elements of the problem. As Figs 3 \& 4 show, different HR metrics have different isopleth profiles, and the importance of these may differ depending on the nature of the problem, the nature of the data set and the subjective importance attached to over- or underestimating HR needs and core areas (e.g. the relative importance of different error profiles may be quite different for academic ecology and endangered species management, respectively).

We found a clear separation between methods that are based on MCPs and those that use density-based measures. The ClustHR algorithm performed adequately on the 'classical' buffalo data sets, but poorly on the less contiguous duck data. The generally weaker performance of the LoCoH algorithm in the analysis, relative to that of other metrics, conflicts with previously published results (Getz \& Wilmers, 2004; Getz et al. 2007). The difference in our conclusions relative to those of Getz \& Wilmers (2004) can be explained by three main points: (1) Getz \& Wilmers focused on a single type of error at a time, rather than the trade-off between errors; (2) Getz \& Wilmers focused heavily on 'difficult' data sets with holes and hard boundaries; and (3) although the LoCoH algorithm starts with a smaller 95\% isopleth, as 


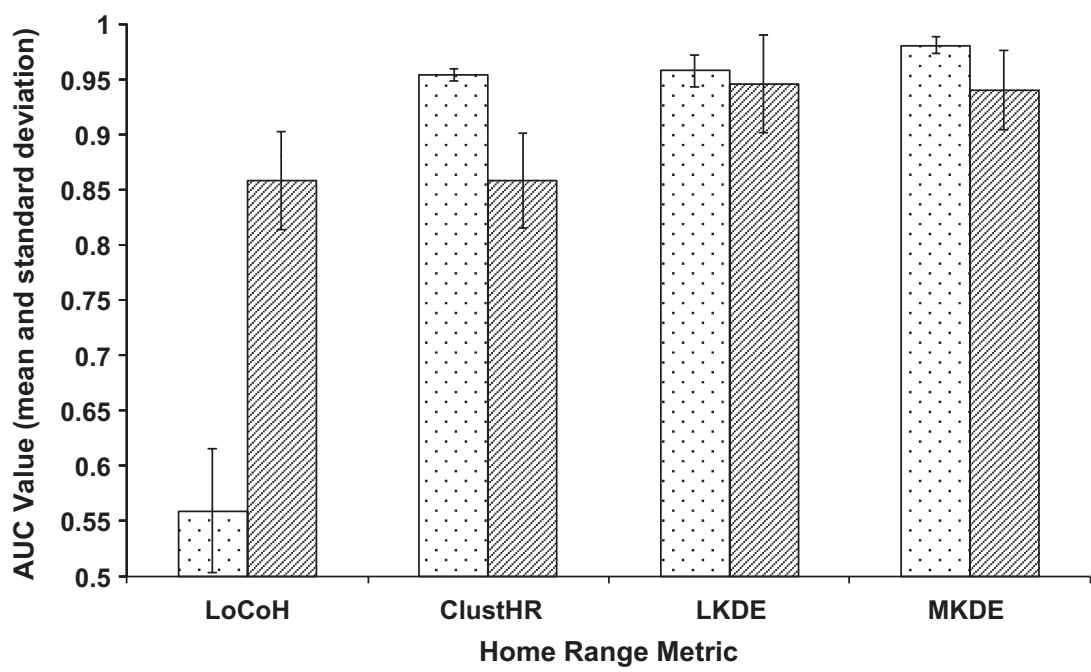

Figure 2 Bar chart showing mean \pm standard deviation of area under the curve (AUC) values for different home range estimators, calculated using GPS telemetry data sets for nine individual African buffaloes (stippled bars, to the left) and 44 individual ducks of two species (cross-hatched bars, to the right).

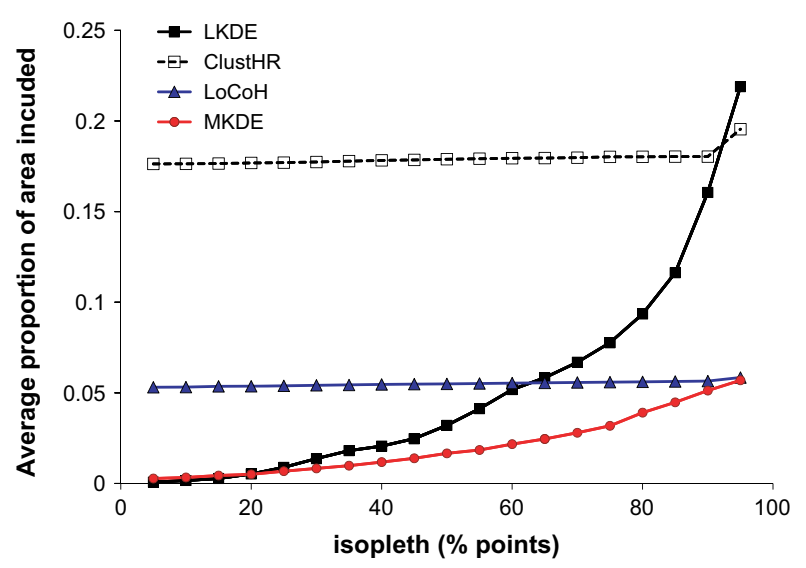

Figure 3 Average proportion of total extent of area under analysis [i.e. entire grid, not minimum convex polygon (MCP)] included by each estimator at each isopleth for 44 different birds (except for CLUST, for which $n=25$; and movement-based kernel density eEstimator (MKDE), for which $n=38$ ). Isopleth areas decline with increasing values in all cases. Note that as this analysis was undertaken using raster data at 0.005 degrees, the value for a given isopleth can not be smaller than the multiple of 0.005 by the total number of grid cells in which any part of an isopleth falls. The relatively flat area profiles of the curves for LoCoH and ClustHR reflect both their initially rapid exclusion of points and their tendency to maintain at least some isopleth area in each cluster of points as isopleth values decline. By contrast, the kernel methods drop entire clusters of points at lower isopleth values, resulting in a better trade-off in error rates.

displayed in Fig. 3, its HR contours show less variation and contract less steeply than the kernel and distance-based metrics, yielding a lower AUC across its full profile.

Claims made about the MKDE estimator (Benhamou \& Cornelis, 2010) were supported, with the MKDE metric producing a more parsimonious HR estimate than the LKDE at each isopleth. The buffalo data presumably show this distinction more strongly because the mechanistic assumptions underlying the MKDE are supported for buffalo but not for ducks. It is important to note, however, that greater parsimony in isopleth areas does not necessarily translate into a better trade-off in error rates; consideration only of isopleth areas ignores type II errors, and the MKDE estimator may suffer in AUC comparisons where there are numerous observations per unit of analysis (grid cell) by weighting the centres of the HR too highly and consequently suffering from inflated type II errors.

In general, methods that use MCPs (such as LoCoH, ClustHR, and simple MCPs) do not appear to be statistically defensible and should be avoided unless there is a strong ecological or conservation-related reason why they should be used. They are also relatively slow in current implementations in R. Studies that have applied these methods appropriately are by no means invalidated by the results presented here, and all of the estimators do vastly better than a random classifier, but there is no obvious reason to keep using a method that is statistically and computationally weaker and offers no additional biological insights.

Among kernel density-based measures and other less widely used alternatives, such as distance-based metrics, the central challenge is to find the best combination of statistical rigour and biological meaning. Kernel density estimation is not necessarily the strongest approach in all situations, particularly with small data sets and where there is uncertainty over parameter choices (Hemson et al., 2005), although the AUC can be used to select optimal parameters. The LKDE estimator, as implemented by Calenge (2006) in the Kernel UD routine of the $\mathrm{R}$ package adehabitat, did, however, emerge from our analysis as a strong candidate for use as a standard model in tracking studies. Under conditions in which resightings are relatively far apart relative to the rate of movement of the animal, as for our bird data, the additional insights offered by the MKDE appear to add little to the statistical accuracy of the LKDE. However, for data sets in which frequent resightings (relative to the scale of movement of the animal) are available, MKDE approaches offer a 
(a)

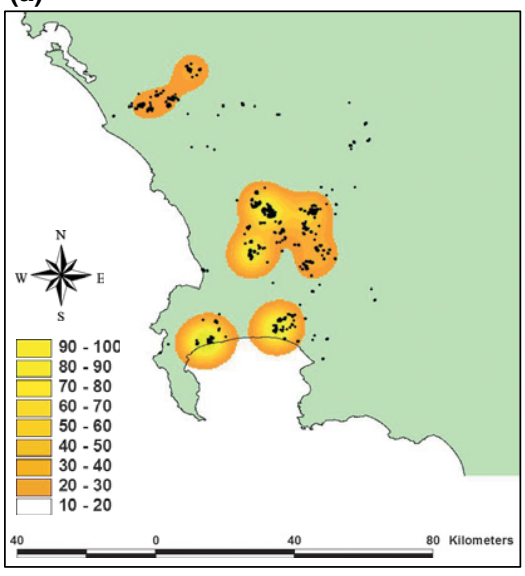

(b)

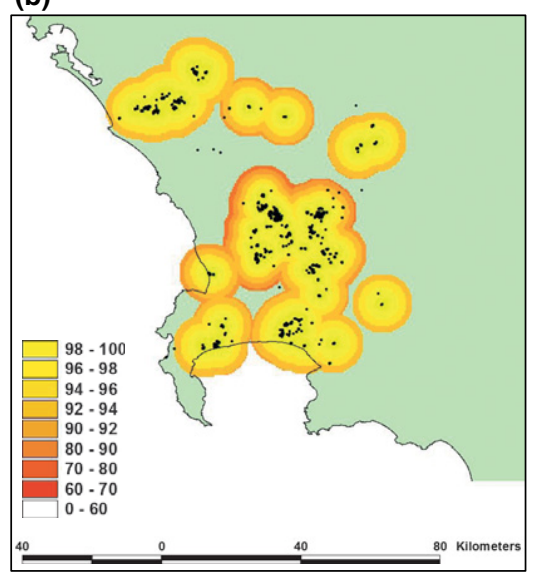

(c)

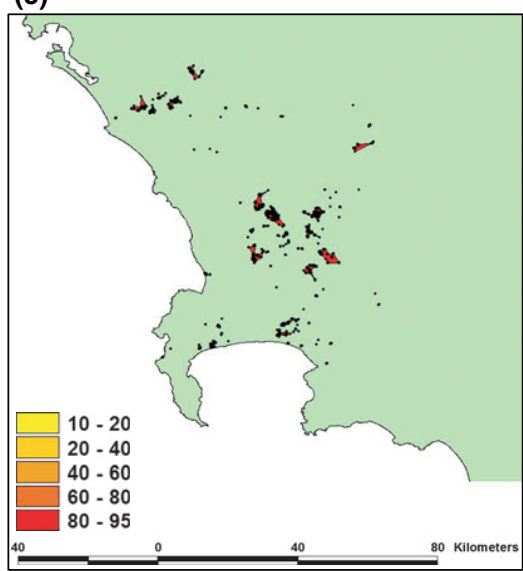

Figure 4 Examples of differences in apparent cluster structure of the home range of Egyptian Goose 77135, as estimated using (a) LKDE, (b) movement-based kernel density eEstimator (MKDE) and (c) LoCoH. Black dots indicate locations at which bird was recorded; shading indicates isopleth values. This home range shows clear evidence of a hierarchical, multi-scale structure.

Table 1 Summary of subjective recommendations for the use of home range metrics considered in this paper

\begin{tabular}{|c|c|c|c|c|}
\hline Property of estimator & ClustHR & $\mathrm{LoCoH}$ & LKDE & MKDE \\
\hline $\begin{array}{l}\text { Trade-off omission/ } \\
\text { commission (AUC) }\end{array}$ & : : & : : & (:) & (:) \\
\hline Biological foundation & 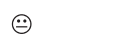 & $\oplus$ & (:) & (:) \\
\hline Mechanistic foundation & (:) & (:) & (:) & (:) \\
\hline Speed of implementation & (:) & : : & ;) & ;) \\
\hline Risk of overfitting & ;) & ;) & (:) & $\odot$ \\
\hline $\begin{array}{l}\text { Integration of physical } \\
\text { barriers }\end{array}$ & 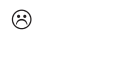 & ;) & : : & (:) \\
\hline $\begin{array}{l}\text { Integration of activity sensor } \\
\text { data }\end{array}$ & (2) & : : & (:) & (:) \\
\hline
\end{tabular}

AUC, area under the curve; MKDE, movement-based kernel density eEstimator.

theoretically sound and biologically meaningful way of finetuning HR estimates and improving on LKDE.

None of the HR metrics that we have considered in this analysis is truly multi-scale (e.g. Marzluff et al., 2004). Cross-scale dynamics are important drivers of ecosystem pattern (Peters et al., 2007) and understanding multi-scale impacts on organisms remains an important challenge for conservation biology. The development of HR models that can be used to capture the combined impacts of broad-scale landscape structure and fine-scale habitat heterogeneity on animal populations is a highly desirable goal. One approach to developing a multi-scale HR estimate would be to cluster points into sequentially larger groups with an algorithm like DBScan (Ester et al., 1996) or its hierarchical cousin, OPTICS (Ankerst et al., 1999), and then run a kernel density estimator on clusters of points individually to build up a composite HR estimate in which each point in a data set can belong to multiple hierarchical levels.
Linking individual-, population- and species-level models is an equally important challenge for conservation biogeography. If the hypothesized mechanisms driving species occurrences in a given case are correct, hierarchical models should be consistent across different scales and levels of analysis (Cumming, 2007). Home range metrics should thus offer a tool for cross-validation and hypothesis testing (e.g., Trivedi et al., 2008; Randin et al., 2009). In theory, it should be possible to combine mechanistic analyses of movement paths and activity patterns (Jonsen et al., 2003) with hierarchical HR estimators to understand and explore multi-scale patterns of habitat use and their interaction with multiple scales of environmental variation. For example, in the same way that Cumming \& Van Vuuren (2006) stacked individual species models to explore the potential impacts of climate change on acarine biodiversity, it should be possible to stack individual HR estimates to explore the strengths and weaknesses of species occurrence models. Where such comparisons use comparable data sets (i.e. equivalent grain, extent, and prevalence), the approach that we have presented, by virtue of its applicability to both $\mathrm{HR}$ estimates and species occurrence models, has the potential to be used as a way of comparing different models and for fine-tuning parameter choices.

\section{ACKNOWLEDGEMENTS}

We are grateful to Simon Benhamou for assistance in running the MKDE estimator and for his advice and encouragement during the preparation of this paper. Useful comments were also provided by Nicolas Gaidet, Ingolf Kühn and two anonymous referees. GC thanks Cirad, the Oppenheimer Foundation and the University of Cape Town for financial assistance during the writing period. Satellite telemetry on African buffalo was funded by the French National Research Agency (ANR Mobility project, West Africa), the European Union 
(Parsel project, Zimbabwe) and the French Foreign Ministry through the French Embassy in Zimbabwe (RP-PCP Grant 2008). Satellite telemetry on ducks was funded by USAID through a subcontract from the Wildlife Conservation Society's Global Avian Influenza Network for Surveillance (GAINS) programme, with additional support from the DST/ NRF Centre of Excellence at the Percy FitzPatrick Institute.

\section{REFERENCES}

Akcakaya, H.R. (2000) Viability analyses with habitat-based metapopulation models. Population Ecology, 42, 45-53.

Ankerst, M., Breunig, M., Kriegel, H. \& Sander, J. (1999) Optics: ordering points to identify the clustering structure. ACM SIGMOD International Conference on Management of Data (ed. by A. Delis, C. Faloutsos and S. Ghandeharizadeh), pp. 49-60 Philadelphia, PA: ACM Press.

Benhamou, S. (2011) Dynamic approach to space and habitat use based on biased random bridges. PLoS ONE, 6, e14592. doi:10.1371/journal.pone.0014592

Benhamou, S. \& Cornelis, D. (2010) Incorporating movement behavior and barriers to improve biological relevance of Kernel home range space use estimates. The Journal of Wildlife Management, 74, 1353-1360.

Börger, L., Dalziel, B.D. \& Fryxell, J.M. (2008) Are there general mechanisms of animal home range behaviour? A review and prospects for future research. Ecology Letters, 11, 637-650.

Boyce, M. (2006) Scale for resource selection functions. Diversity and Distributions, 12, 269-276.

Burgman, M. \& Fox, J. (2003) Bias in species range estimates from minimum convex polygons: implications for conservation and options for improved planning. Animal Conservation, 6, 19-28.

Calenge, C. (2006) The package "adehabitat" for the R software: a tool for the analysis of space and habitat use by animals. Ecological Modelling, 197, 516-519.

Cumming, G.S. (2000) Using between-model comparisons to fine-tune linear models of species ranges. Journal of Biogeography, 27, 441-455.

Cumming, G.S. (2002) Comparing climate and vegetation as limiting factors for species ranges of African ticks. Ecology, 83, 255-268.

Cumming, G.S. (2007) Global biodiversity scenarios and landscape ecology. Landscape Ecology, 22, 671-685.

Cumming, G.S. (2009) Current themes and recent advances in modelling species occurrences. f1000 Biology Reports, 1, 94. (see http://f1000.com/reports/b/1/94/).

Cumming, G.S. \& Van Vuuren, D.P. (2006) Will climate change affect ectoparasite species ranges? Global Ecology and Biogeography, 15, 486-497.

Ester, M., Kriegel, H., Sander, J. \& Xu, X. (1996) A densitybased algorithm for discovering clusters in large spatial databases with noise. Second International Conference on Knowledge Discovery and Data Mining (KDD-96). (ed. by E. Simoudis, J. Han and U. Fayyad), pp. 226-231. AAAI Press, Menlo Alto, CA.
Fielding, A.H. \& Bell, J.F. (1997) A review of methods for the assessment of prediction errors in conservation presence/absence models. Environmental Conservation, 24, 3849.

Franklin, J. (2010) Mapping species distributions: spatial inference and prediction. Cambridge University Press, Cambridge.

Getz, W. \& Wilmers, C. (2004) A local nearest-neighbor convex-hull construction of home ranges and utilization distributions. Ecography, 27, 489-505.

Getz, W.M., Fortmann-Roe, S., Cross, P.C., Lyons, A.J., Ryan, S.J., Wilmers, C.C. \& Coulson, T. (2007) LoCoH: nonparameteric kernel methods for constructing home ranges and utilization distributions. PLOS ONE, 2, e207.

Guisan, A., Lehmann, A., Ferrier, S., Austin, M., Overton, J.M.C., Aspinall, R. \& Hastie, T. (2006) Making better biogeographical predictions of species' distributions. Journal of Applied Ecology, 43, 386-392.

Hemson, G., Johnson, P., South, A., Kenward, R., Ripley, R. \& MacDonald, D. (2005) Are kernels the mustard? Data from global positioning system (GPS) collars suggests problems for kernel home-range analyses with least squares crossvalidation. Journal of Animal Ecology, 74, 455-463.

Horne, J.S. \& Garton, E.O. (2006) Selecting the best home range model: an information-theoretic approach. Ecology, 87, 1146-1152.

Jonsen, I.D., Myers, R.A. \& Flemming, J.M. (2003) Metaanalysis of animal movement using state-space models. Ecology, 84, 3055-3063.

Kelt, D.A. \& Van Vuren, D.H. (2001) The ecology and macro-ecology of mammalian home range area. The American Naturalist, 157, 637-645.

Kenward, R., Clarke, R., Hodder, K. \& Walls, S. (2001) Density and linkage estimators of home range: nearest-neighbor clustering defines multinuclear cores. Ecology, 82, 19051920.

Kie, J.G., Matthiopoulos, J., Fieberg, J., Powell, R.A., Cagnacci, F., Mitchell, M.S., Gaillard, J.M. \& Moorcroft, P.R. (2010) The home-range concept: are traditional estimators still relevant with modern telemetry technology? Philosophical Transactions of the Royal Society B: Biological Sciences, 365, 2221-2231.

Laver, P. \& Kelly, M. (2008) A critical review of home range studies. The Journal of Wildlife Management, 72, 290-298.

Lichti, N.I. \& Swihart, R.K. (2011) Estimating utilization distributions with kernel versus local convex hull methods. The Journal of Wildlife Management, 75, 413-422.

Marzluff, J.M., Millspaugh, J.J., Hurvitz, P. \& Handcock, M.S. (2004) Relating resources to a probabilistic measure of space use: forest fragments and steller's jays. Ecology, 85, 1411-1427.

Mayor, S.J., Schneider, D.C., Schaefer, J.A. \& Mahoney, S.P. (2009) Habitat selection at multiple scales. Ecoscience, 16, 238-247.

Mohr, C. (1947) Table of equivalent populations of North American small mammals. American Midland Naturalist, 37, 223-249. 
Peters, D.P.C., Bestelmeyer, B.T. \& Turner, M.G. (2007) Cross-scale interactions and changing pattern-process relationships: consequences for system dynamics. Ecosystems, 10, 790-796.

Peterson, A.T., Papes, M. \& Soberon, J. (2008) Rethinking receiver operating characteristic analysis applications in ecological niche modeling. Ecological Modelling, 213, 63-72.

Randin, C.F., Engler, R., Normand, S., Zappa, M., Zimmermann, N.E., Pearman, P.B., Vittoz, P., Thuiller, W. \& Guisan, A. (2009) Climate change and plant distribution: local models predict high-elevation persistence. Global Change Biology, 15, 1557-1569.

Richardson, D.M. \& Whittaker, R.J. (2010) Conservation biogeography - foundations, concepts and challenges. Diversity and Distributions, 16, 313-320.

Schoener, T.W. (1981) An empirically based estimate of home range. Theoretical Population Biology, 20, 281-325.

Seaman, D., Millspaugh, J., Kernohan, B., Brundige, G., Raedeke, K. \& Gitzen, R. (1999) Effects of sample size on kernel home range estimates. The Journal of Wildlife Management, 63, 739-747.

Thomas, D.L. \& Taylor, E.J. (2006) Study designs and tests for comparing resource use and availability II. The Journal of Wildlife Management, 70, 324-336.

Trivedi, M.R., Berry, P.M., Morecroft, M.D. \& Dawson, T.P. (2008) Spatial scale affects bioclimate model projections of climate change impacts on mountain plants. Global Change Biology, 14, 1089-1103.

Valeix, M., Loveridge, A.J., Chamaille-Jammes, S., Davidson, Z., Murindagomo, F., Fritz, H. \& Macdonald, D.W. (2009) Behavioral adjustments of African herbivores to predation risk by lions: spatiotemporal variations influence habitat use. Ecology, 90, 23-30.

Vaughan, I.P. \& Ormerod, S.J. (2005) The continuing challenges of testing species distribution models. Journal of Applied Ecology, 42, 720-730.

Worton, B. (1989) Kernel methods for estimating the utilization distribution in home-range studies. Ecology, 70, 164-168.

Worton, B. (1995) Using Monte Carlo simulation to evaluate kernel-based home range estimators. The Journal of Wildlife Management, 59, 794-800.

\section{SUPPORTING INFORMATION}

Additional Supporting Information may be found in the online version of this article:
Appendix S1 Confusion matrix, or error matrix, for a home range estimate.

Appendix S2 Home range metrics that were compared, their computational basis and details of the specific implementation used in the comparison.

Appendix S3 Details of species, transmitters and sample sizes for each individual data set used in this analysis.

Appendix S4 Examples of Mat' and R code used in the analysis.

Appendix S5 Details of implementation of analyses for duck and buffalo data sets, respectively, and running speeds for different algorithms.

As a service to our authors and readers, this journal provides supporting information supplied by the authors. Such materials are peer-reviewed and may be re-organized for online delivery, but are not copy-edited or typeset. Technical support issues arising from supporting information (other than missing files) should be addressed to the authors.

\section{BIOSKETCHES}

Graeme Cumming is a landscape ecologist and conservation biologist. He runs a far-ranging research programme on the influence of spatial heterogeneity on ecological and human systems and recently wrote a book about spatial resilience.

Daniel Cornélis is interested in the emerging discipline of movement ecology. His work focuses on the mobility of wild ungulates (conservation ecology) and the spatial interactions between wild and domestic animals (health ecology, conflicts for space and resources). His current focus is on using GPS tracking tools and new models of analysis to investigate the processes underlying the spatial distribution and movements of large African mammals.

Author contributions: G.S.C. conceived the study, analysed the duck data, and led the writing of the paper. D.C. helped to develop the concepts, analysed the buffalo data, and co-wrote the paper.

Editor: Ingolf Kuhn 